\title{
Article
}

\section{On Maximal Distance Energy}

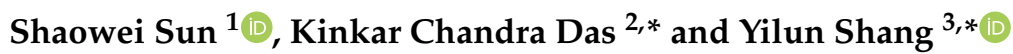 \\ 1 School of Science, Zhejiang University of Science and Technology, Hangzhou 310023, China; \\ sunshaowei2009@126.com \\ 2 Department of Mathematics, Sungkyunkwan University, Suwon 16419, Korea \\ 3 Department of Computer and Information Sciences, Northumbria University, Newcastle NE1 8ST, UK \\ * Correspondence: kinkardas2003@gmail.com (K.C.D.); yilun.shang@northumbria.ac.uk (Y.S.)
}

check for

updates

Citation: Sun, S.; Das, K.C.; Shang, Y. On Maximal Distance Energy. Mathematics 2021, 9, 360. https:// doi.org/10.3390/math9040360

Academic Editor: Pavel Trojovský, Diego Marques and Iwona Włoch

Received: 19 January 2021

Accepted: 9 February 2021

Published: 11 February 2021

Publisher's Note: MDPI stays neutral with regard to jurisdictional claims in published maps and institutional affiliations.

\begin{abstract}
Let $G$ be a graph of order $n$. If the maximal connected subgraph of $G$ has no cut vertex then it is called a block. If each block of graph $G$ is a clique then $G$ is called clique tree. The distance energy $E_{D}(G)$ of graph $G$ is the sum of the absolute values of the eigenvalues of the distance matrix $D(G)$. In this paper, we study the properties on the eigencomponents corresponding to the distance spectral radius of some special class of clique trees. Using this result we characterize a graph which gives the maximum distance spectral radius among all clique trees of order $n$ with $k$ cliques. From this result, we confirm a conjecture on the maximum distance energy, which was given in Lin et al. Linear Algebra Appl 467(2015) 29-39.
\end{abstract}

Keywords: distance matrix; distance spectral radius; distance energy

MSC: $05 C 50$

\section{Introduction}

Let $G$ be a connected graph with $V(G)=\left\{v_{1}, v_{2}, \ldots, v_{n}\right\}$ and edge set $E(G)$, where $|V(G)|=n$. For $v_{i}, v_{j} \in V(G), d_{i, j}$ denotes the distance between vertices $v_{i}$ and $v_{j}$. In particular, $d_{i, i}=0$ for any $v_{i} \in V(G)$. Let $D(G)=\left(d_{i, j}\right)_{n \times n}$ be the distance matrix of graph $G$. Let $\lambda_{i}(1 \leq i \leq n)$ denote the eigenvalues of $D(G)$ with non-increasing order. Also let $\lambda\left(=\lambda_{1}\right)$ be the distance spectral radius of a graph $G$. When more than one graph is under consideration, then we write $\lambda_{i}(G)$ instead of $\lambda_{i}$. By the Perron-Frobenius theorem, a unique unit eigenvector $\mathbf{x}$ corresponding to the largest distance eigenvalue of $G$ has all positive eigencomponents, which is called the Perron vector of $D(G)$. Several studies on this topic have been conducted, see [1-7] and the survey [8].

The energy of a graph $G$, often denoted by $\mathcal{E}(G)$, is defined to be the sum of the absolute value of the eigenvalues of its adjacency matrix of a graph. The energy of a graph was first defined by Ivan Gutman in 1978 [9]. However, the motivation for his definition appeared much earlier, in the 1930's, when Erich Hückel proposed the famous Hückel Molecular Orbital Theory. Hückel's method allows chemists to approximate energies associated with $\pi$-electron orbitals in a special class of molecules called conjugated hydrocarbons. From the motivation of graph energy, Indulal et al. [10] proposed the distance energy $E_{D}(G)$ of $G$ which is defined by

$$
E_{D}=E_{D}(G)=\sum_{i=1}^{n} \lambda_{i}
$$

Since the trace of $D(G)$ is zero, we have

$$
E_{D}=E_{D}(G)=2 \sum_{\lambda_{i}>0} \lambda_{i}=-2 \sum_{\lambda_{i}<0} \lambda_{i}
$$


Extremal graph theory is one of the important topics in graph theory and combinatorics. In extremal graph theory to find the extremal (maximal and/or minimal) graphs for some graph invariant is the important and interesting problem. It is very difficult to find the exact value of the distance energy of graphs. In this paper we are concentrating to obtain the maximal distance energy for some special class of graphs. For the basic mathematical properties of $E_{D}(G)$, including various lower and upper bounds, see [8,11-14] and the references therein.

If the maximal connected subgraph of $G$ has no cut vertex then it is called a block. If each block of graph $G$ is a clique then $G$ is called clique tree. Let $P_{k+1}$ be a path graph of order $k+1$. A clique path, denoted by $\mathbb{P}_{n_{1}, n_{2}, \ldots, n_{k}}$, is a graph which is obtained from $P_{k+1}$ by replacing each edge of $P_{k+1}$ by a clique $K_{n_{i}}\left(n_{i} \geq 2\right)$ such that $V\left(K_{n_{i}}\right) \cap V\left(K_{n_{j}}\right)=\varnothing$ for $j \neq$ $i-1, i+1$ and $2 \leq i \leq k-1$. If $n_{s+1}=n_{s+2}=\cdots=n_{s+t}$, we use $\mathbb{P}_{n_{1}, \ldots, n_{s}, n_{s+1} \cdot t, n_{s+t+1}, \ldots, n_{k}}$ to denote $\mathbb{P}_{n_{1}, n_{2}, \ldots, n_{k}}$ for short.

Lin et al. [15] discussed several properties of clique trees and discovered that the positive inertia and the negative inertia of the distance matrix of a clique tree with $n$ vertices are 1 and $n-1$, respectively. Among all clique trees with order $n$, the graph with the minimum distance energy has been characterized in [15]. They also gave a conjecture related to the maximum distance energy as follows:

Conjecture 1 ([15]). The graph $\mathbb{P}_{\left\lceil\frac{n-k+3}{2}\right\rceil, 2 \cdot(k-2),\left\lfloor\frac{n-k+3}{2}\right\rfloor}$ gives the maximum distance energy among all clique trees with cliques $K_{n_{1}}, \ldots, K_{n_{k}}$ and order $n$.

Due to the fact that any clique tree has exactly one positive distance eigenvalue, then Conjecture 1 is equivalent to the following:

Conjecture 2. The graph $\mathbb{P}_{\left\lceil\frac{n-k+3}{2}\right\rceil, 2 \cdot(k-2),\left\lfloor\frac{n-k+3}{2}\right\rfloor}$ gives the maximum largest distance eigenvalue among all clique trees with cliques $K_{n_{1}}, \ldots, K_{n_{k}}^{2}$ and order $n$.

Please note that Conjecture 2 has been proved for $k=3$ [4]. In the next section, we will confirm that Conjecture 2 holds for the remaining cases and thus Conjecture 1 follows immediately.

\section{Proof of Conjecture 2}

In [15], the authors revealed that the clique trees with the maximum spectral radius belong to a special class of clique paths.

Lemma 1 ([15]). The graph $\mathbb{P}_{s, 2 \cdot(k-2), t}$ with respect to the restriction $s+t=n-k+3$ gives the maximum largest distance eigenvalue among all clique trees with cliques $K_{n_{1}}, \ldots, K_{n_{k}}$ and order $n$.

By Lemma 1, for proving Conjecture 2, we only need to find the maximum distance spectral radius of $\mathbb{P}_{s, 2 \cdot(k-2), t}$, where $s+t=n-k+3$. Please note that Conjecture 2 follows directly if $k=n-1$. For $k=n-2$, we have $s+t=5$ and hence $\mathbb{P}_{3,2 \cdot(n-3)}$ gives the maximum distance spectral radius among all clique trees with cliques $K_{n_{1}}, \ldots, K_{n_{k}}$ and order $n$, which provide the Conjecture 2 is true. Therefore, hereafter we only consider the cases with $4 \leq k \leq n-3$. Now let $\mathbb{P}(n, k, s, t) \cong \mathbb{P}_{s, 2 \cdot(k-2), t}$ with $s+t=n-k+3$, whose vertex set is $V(\mathbb{P}(n, k, s, t))=\left\{v_{1}, v_{2}, \ldots, v_{n}\right\}$ and edge set is $E(\mathbb{P}(n, k, s, t))=$ $E_{1} \cup E_{2} \cup E_{3}$, where $E_{1}=\left\{v_{i} v_{j} \mid 1 \leq i<j \leq s\right\}, E_{2}=\left\{v_{i} v_{j} \mid n-t+1 \leq i<j \leq n\right\}$ and $E_{3}=\left\{v_{i} v_{i+1} \mid s \leq i \leq n-t\right\}$. Let $\mathbf{x}^{T}=\left(x_{v_{1}}, x_{v_{2}}, \ldots, x_{v_{n}}\right)$ be the unit positive eigenvector corresponding to spectral radius $\lambda$ of $D(\mathbb{P}(n, k, s, t))$. By symmetry, one can see that $x_{v_{1}}=x_{v_{2}}=\cdots=x_{v_{s-1}}$ and $x_{v_{n-t+2}}=x_{v_{n-t+3}}=\cdots=x_{v_{n}}$. Thus, we may suppose that

$$
\mathbf{x}^{T}=(\underbrace{x_{1}, \ldots, x_{1}}_{s-1}, x_{2}, x_{3}, \ldots, x_{k}, \underbrace{x_{k+1}, \ldots, x_{k+1}}_{t-1}) .
$$


From $D(\mathbb{P}(n, k, s, t)) \mathbf{x}=\lambda \mathbf{x}$

$$
\left\{\begin{array}{l}
\lambda x_{1}=(s-2) x_{1}+k(t-1) x_{k+1}+\sum_{j=2}^{k}(j-1) x_{j} \\
\lambda x_{i}=(s-1)(i-1) x_{1}+(k-i+1)(t-1) x_{k+1}+\sum_{\substack{j=2 \\
j \neq i}}^{k}|j-i| x_{j}, i=2,3, \ldots, k \\
\lambda x_{k+1}=k(s-1) x_{1}+(t-2) x_{k+1}+\sum_{j=2}^{k}(k-j+1) x_{j} .
\end{array}\right.
$$

First, we discuss the properties of Perron vector of $D(\mathbb{P}(n, k, s, t))$.

Lemma 2. Let $G \cong \mathbb{P}(n, k, s, t)$ with $k=2 p-2$ ( $p$ is an integer, $p \geq 3$ ) and $s \geq t+2$. Let

$$
\mathbf{x}^{T}=(\underbrace{x_{1}, \ldots, x_{1}}_{s-1}, x_{2}, x_{3}, \ldots, x_{k}, \underbrace{x_{k+1}, \ldots, x_{k+1}}_{t-1})
$$

be the Perron vector of $D(G)$. Then $x_{p+i}>x_{p-i}$ for $1 \leq i \leq p-2$. Moreover, if $p \geq 4$, then

$$
x_{p+i}-x_{p-i}>x_{p+i-1}-x_{p-i+1} \text { for } 2 \leq i \leq p-2 .
$$

Proof. Since $k=2 p-2$, from (1), we obtain

$$
\begin{aligned}
& \lambda\left(x_{2 p-1}-x_{1}\right)=(k s-k-s+2) x_{1}-(t k-k-t+2) x_{2 p-1}+2 \sum_{j=1}^{p-2} j\left(x_{p-j}-x_{p+j}\right), \\
& \lambda\left(x_{p+1}-x_{p-1}\right)=2\left((s-1) x_{1}-(t-1) x_{2 p-1}+\sum_{j=1}^{p-2}\left(x_{p-j}-x_{p+j}\right)\right)
\end{aligned}
$$

and if $p \geq 4$,

$$
\lambda\left(x_{p+i}-x_{p-i}\right)=2 i\left((s-1) x_{1}-(t-1) x_{2 p-1}+\sum_{j=i}^{p-2}\left(x_{p-j}-x_{p+j}\right)\right)+2 \sum_{j=1}^{i-1} j\left(x_{p-j}-x_{p+j}\right),
$$

where $i=2,3, \ldots, p-2$. Setting $x_{p+i}-x_{p-i}=y_{i}(1 \leq i \leq p-1)$ in the above, we get

$$
\begin{aligned}
& \lambda y_{p-1}=(k s-k-s+2) x_{1}-(t k-k-t+2) x_{2 p-1}-2 \sum_{j=1}^{p-2} j y_{j}, \\
& \lambda y_{1}=2\left((s-1) x_{1}-(t-1) x_{2 p-1}-\sum_{j=1}^{p-2} y_{j}\right),
\end{aligned}
$$

and if $p \geq 4$,

$$
\lambda y_{i}=2 i\left((s-1) x_{1}-(t-1) x_{2 p-1}-\sum_{j=i}^{p-2} y_{j}\right)-2 \sum_{j=1}^{i-1} j y_{j}
$$

where $i=2,3, \ldots, p-2$. Combining (2) and setting $i=p-2$ in (4) with $k=2 p-2$, we have

$$
\begin{aligned}
& \lambda y_{p-1}-\lambda y_{p-2} \\
= & (k s-k-s+2) x_{1}-(t k-k-t+2) x_{2 p-1}-2(p-2)\left((s-1) x_{1}-(t-1) x_{2 p-1}\right) \\
= & s x_{1}-t x_{2 p-1}
\end{aligned}
$$


that is,

$$
\lambda y_{p-2}=(\lambda+t) x_{2 p-1}-(\lambda+s) x_{1}
$$

as $y_{p-1}=x_{2 p-1}-x_{1}$.

Using (3), from (4),

$$
\begin{aligned}
\lambda y_{i}-\lambda y_{i-1} & =2\left((s-1) x_{1}-(t-1) x_{2 p-1}-\sum_{j=i}^{p-2} y_{j}\right) \\
& =2\left((s-1) x_{1}-(t-1) x_{2 p-1}-\sum_{j=1}^{p-2} y_{j}\right)+2 \sum_{j=1}^{i-1} y_{j} \\
& =\lambda y_{1}+2 \sum_{j=1}^{i-1} y_{j}
\end{aligned}
$$

that is,

$$
y_{i}=y_{1}+\frac{2}{\lambda} \sum_{j=1}^{i-1} y_{j}+y_{i-1}, i=2,3, \ldots, p-2 .
$$

From (6), we conclude that if $y_{1}$ is positive (zero or negative) then all $y_{i}(i=2,3, \ldots$, $p-2$ ) are positive (zero or negative, respectively).

Claim 1. $y_{1}>0$.

Proof of Claim 1. We suppose that $y_{1} \leq 0$. This implies that $y_{i} \leq 0(i=2,3, \ldots, p-2)$, thus

$$
\sum_{j=1}^{p-2} y_{j} \leq 0
$$

Using $y_{p-2} \leq 0$ in (5), we obtain that

$$
x_{1} \geq \frac{\lambda+t}{\lambda+s} x_{2 p-1}
$$

It implies that

$$
(s-1) x_{1}-(t-1) x_{2 p-1} \geq \frac{(s-t)(\lambda+1)}{\lambda+s} x_{2 p-1}>0,
$$

as $s>t, \lambda>0$ and $x_{2 p-1}>0$. Combining the last inequality with (3), we conclude that $y_{1}>0$, which is a contradiction. We finish the proof of Claim 1.

Using Claim 1, we conclude that $y_{i}>0$, i.e., $x_{p+i}>x_{p-i}$ for $1 \leq i \leq p-2$. From (6), we obtain $y_{i}>y_{i-1}$, i.e., $x_{p+i}-x_{p-i}>x_{p+i-1}-x_{p-i+1}$ for $i=2,3, \ldots, p-2$.

By the same method as the above, we also obtain the similar result for odd $k$.

Lemma 3. Let $G \cong \mathbb{P}(n, k, s, t)$ with $k=2 p-1$ ( $p$ is an integer, $p \geq 3$ ) and $s \geq t+2$. Let

$$
\mathbf{x}^{T}=(\underbrace{x_{1}, \ldots, x_{1}}_{s-1}, x_{2}, x_{3}, \ldots, x_{k}, \underbrace{x_{k+1}, \ldots, x_{k+1}}_{t-1})
$$

denote the Perron vector of $D(G)$. Then $x_{p+i}-x_{p+1-i}>x_{p-1+i}-x_{p+2-i}>0$ for $2 \leq i$ $\leq p-1$.

Combining Lemmas 2 and 3, the next theorem follows immediately. 
Theorem 1. Let $G \cong \mathbb{P}(n, k, s, t)$ with $s+t=n-k+3$ and $s \geq t+2$. Let

$$
\mathbf{x}^{T}=(\underbrace{x_{1}, \ldots, x_{1}}_{s-1}, x_{2}, x_{3}, \ldots, x_{k}, \underbrace{x_{k+1}, \ldots, x_{k+1}}_{t-1})
$$

be Perron vector of $D(G)$. If $k=4$, then $x_{k}-x_{2}>0$; otherwise, $x_{k+1-i}-x_{i+1}>x_{k-i}-x_{i+2}>0$ for $1 \leq i \leq\left\lfloor\frac{k-3}{2}\right\rfloor$.

Theorem 2. For any pair of integers $s$ and $t$ with $s \geq t+2 \geq 4$,

$$
\lambda_{1}\left(\mathbb{P}_{s, 2 \cdot(k-2), t}\right)<\lambda_{1}\left(\mathbb{P}_{s-1,2 \cdot(k-2), t+1}\right) .
$$

Proof. For convenience, let $G \cong \mathbb{P}_{s, 2 \cdot(k-2), t}$ and let $G^{\prime} \cong \mathbb{P}_{s-1,2 \cdot(k-2), t+1}$. Let

$$
\mathbf{x}^{T}=(\underbrace{x_{1}, \ldots, x_{1}}_{s-1}, x_{2}, x_{3}, \ldots, x_{k}, \underbrace{x_{k+1}, \ldots, x_{k+1}}_{t-1})
$$

be the Perron vector corresponding to spectral radius $\lambda$ of $D(G)$. Then

$$
\begin{aligned}
\lambda\left(G^{\prime}\right)-\lambda(G) & \geq \mathbf{x}^{T}\left(D\left(G^{\prime}\right)-D(G)\right) \mathbf{x} \\
& =2 x_{1}\left((k-1)(s-2) x_{1}-(k-1)(t-1) x_{k+1}+\sum_{i=1}^{\left\lfloor\frac{k-1}{2}\right\rfloor}(k-2 i)\left(x_{i+1}-x_{k-i+1}\right)\right) .
\end{aligned}
$$

Now let $F=(k-1)(s-2) x_{1}-(k-1)(t-1) x_{k+1}+F_{1}$, where

$$
F_{1}=\sum_{i=1}^{\left\lfloor\frac{k-1}{2}\right\rfloor}(k-2 i)\left(x_{i+1}-x_{k-i+1}\right) .
$$

To prove $\lambda\left(G^{\prime}\right)>\lambda(G)$, we have to prove that $F>0$. From $D(G) \mathbf{x}=\lambda \mathbf{x}$,

$$
\lambda\left(x_{k+1}-x_{1}\right)=(k s-k-s+2) x_{1}-(k t-k-t+2) x_{k+1}+F_{1}
$$

and

$$
\lambda\left(x_{k}-x_{2}\right)=(k-2)\left((s-1) x_{1}-(t-1) x_{k+1}\right)+F_{1} .
$$

Combining the above two equations with $s>t$, we get

$$
\lambda\left(x_{k+1}-x_{1}\right)-\lambda\left(x_{k}-x_{2}\right)=s x_{1}-t x_{k+1}>t\left(x_{1}-x_{k+1}\right),
$$

that is,

$$
x_{k}-x_{2}<\frac{\lambda+t}{\lambda}\left(x_{k+1}-x_{1}\right)
$$

By Theorem 1 with (8), $x_{k+1}>x_{1}$. We can rewrite (7) as

$$
\lambda\left(x_{k+1}-x_{1}\right)=2 F-F_{1}+(k t-k-t) x_{k+1}-(k s-3 k-s+2) x_{1} .
$$

Using Theorem 1 with $s \geq t+2$ and (8), from the above equation, we obtain 


$$
\begin{aligned}
\lambda\left(x_{k+1}-x_{1}\right) & \leq 2 F-F_{1}+(k t-k-t)\left(x_{k+1}-x_{1}\right) \\
& \leq 2 F+\left(x_{k}-x_{2}\right) \sum_{i=1}^{\left\lfloor\frac{k-1}{2}\right\rfloor}(k-2 i)+(k t-k-t)\left(x_{k+1}-x_{1}\right) \\
& <2 F+\frac{\lambda+t}{\lambda}\left(x_{k+1}-x_{1}\right) \sum_{i=1}^{\left\lfloor\frac{k-1}{2}\right\rfloor}(k-2 i)+(k t-k-t)\left(x_{k+1}-x_{1}\right),
\end{aligned}
$$

that is,

$$
2 F>\left(\lambda+k+t-k t-\frac{\lambda+t}{\lambda} F_{2}\right)\left(x_{k+1}-x_{1}\right),
$$

where

$$
F_{2}=\sum_{i=1}^{\left\lfloor\frac{k-1}{2}\right\rfloor}(k-2 i)= \begin{cases}\frac{(k-1)^{2}}{4} & \text { if } k \text { is odd } \\ \frac{k^{2}-2 k}{4} & \text { if } k \text { is even. }\end{cases}
$$

Claim 2. $\lambda \geq F_{2}+(k-1)(t-1)+s-1$.

Proof of Claim 2. Let

$$
r_{i}=\sum_{j=1, j \neq i}^{n} d_{i, j}
$$

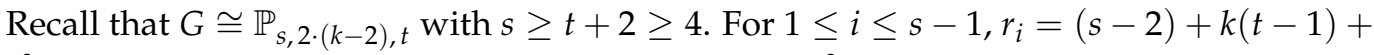
$\frac{k^{2}-k}{2}$. For $n-t+2 \leq i \leq n, r_{i}=(t-2)+k(s-1)+\frac{k^{2}-k}{2}$. For $s \leq i \leq n-t+1$,

$$
r_{i}=(s-1)(i-s+1)+(t-1)(k+s-i-1)+\sum_{j=s, j \neq i}^{n-t+1} d_{i, j} \geq s-1+(k-1)(t-1)+F_{2}
$$

as $s>t$ and

$$
\begin{aligned}
\sum_{j=s, j \neq i}^{n-t+1} d_{i, j} & \geq \begin{cases}2 \sum_{j=1}^{\left\lfloor\frac{k-1}{2}\right\rfloor} j-\frac{k-1}{2} & \text { if } k \text { is odd } \\
2 \sum_{j=1}^{\left\lfloor\frac{k-1}{2}\right\rfloor} j & \text { if } k \text { is even }\end{cases} \\
& =F_{2} .
\end{aligned}
$$

Please note that $r_{1}=r_{2}=\cdots=r_{s-1}<r_{n-t+2}=r_{n-t+3}=\cdots=r_{n}$. Combining the above results, we obtain

$$
\min _{i}\left\{r_{i}\right\} \geq \min \left\{r_{1}, s-1+(k-1)(t-1)+F_{2}\right\}=s-1+(k-1)(t-1)+F_{2} .
$$

It is well-known that $\lambda \geq \min _{i}\left\{r_{i}\right\}$. Thus, we get the result in Claim 2 . By Claim 2, 


$$
\begin{aligned}
& \lambda+k+t-k t-\frac{\lambda+t}{\lambda} F_{2} \\
\geq & F_{2}+(k-1)(t-1)+s-1+k+t-k t-\frac{\lambda+t}{\lambda} F_{2} \\
= & s-\frac{t}{\lambda} F_{2} \\
> & s-t>0 \text { as } \lambda>F_{2} \text { and } s>t .
\end{aligned}
$$

By combining this result with (9), $F>0$. Hence we get the required result.

Remark 1. By Theorem 2, we immediately confirm that Conjecture 2 is true. Hence, the Conjecture 1 is confirmed to be true as well.

Author Contributions: Investigation, S.S., K.C.D., Y.S.; writing-original draft preparation, S.S., K.C.D., Y.S.; writing-review and editing, S.S., K.C.D., Y.S. All authors have read and agreed to the published version of the manuscript.

Funding: This research received no external funding.

Acknowledgments: The authors are very grateful to the reviewers for their constructive comments and suggestions.

Conflicts of Interest: The authors declare no conflict of interest.

\section{References}

1. Das, K.C. Maximal and minimal entry in the principal eigenvector for the distance matrix of a graph. Discret. Math. 2011, 311, 2593-2600. [CrossRef]

2. Das, K.C.; Gutman, I. Upper bounds on distance energy. MATCH Commun. Math. Comput. Chem. 2020, in press.

3. Lin, H.; Das, K.C.; Wu, B. Remoteness and distance eigenvalues of a graph. Discrete Appl. Math. 2016, 215, 218-224. [CrossRef]

4. Lin, H.; Feng, L. The distance spectral radius of graphs with given independence number. Ars Comb. 2015, 121, $113-123$.

5. Lin, H.; Zhou, B. Distance spectral radius of trees with given number of segments. Linear Algebra Appl. 2020, 600, 40-59. [CrossRef]

6. Shang, Y. Bounds of distance Estrada index of graphs. Ars Comb. 2016, 128, 287-294.

7. Zhang, M.; Li, S. Extremal cacti of given matching number with respect to the distance spectral radius. Appl. Math. Comput. 2016, 291, 89-97. [CrossRef]

8. Aouchiche, M.; Hansen, P. Distance spectra of graphs: A survey. Linear Algebra Appl. 2014, 458, 301-386. [CrossRef]

9. Gutman, I. The energy of a graph, 10. In Proceedings of the Steiermärkisches Mathematisches Symposium (Stift Rein, Graz 1978), Graz, Austria, 15-16 November 1978; Volume 103, pp. 1-22.

10. Indulal, G.; Gutman, I.; Vijayakumar, A. On distance energy of graphs. MATCH Commun. Math. Comput. Chem. 2008, 60, 461-472.

11. Alhevaz, A.; Baghipur, M.; Ganie, H.A.; Shang, Y. On the generalized distance energy of graphs. Mathematics 2020, 8, 17. [CrossRef]

12. Ilić, A. Distance spectra and distance energy of integral circulant graphs. Linear Algebra Appl. 2010, 433, 1005-1014. [CrossRef]

13. Sun, S.; Das, K.C. Proof of a conjecture on distance energy change of complete multipartite graph due to edge deletion. Linear Algebra Appl. 2021, 611, 253-259. [CrossRef]

14. Zhou, B.; Ilić, A. On distance spectral radius and distance energy of graphs. MATCH Commun. Math. Comput. Chem. 2010, 64, 261-280.

15. Lin, H.; Liu, R.; Lu, X. The inertia and energy of the distance matrix of a connected graph. Linear Algebra Appl. 2015, 467, 29-39. [CrossRef] 